\title{
Elastic Vector Meson Production at HERA
}

\section{Bruce Mellado* (On behalf of the $\mathbf{H} 1$ and ZEUS Collaborations)}

Columbia University, 538 120th Street West, New York, NY 10027, USA E-mail:

mellado@nevisi.nevis.columbia.edu'

ABstract: The H1 and ZEUS Collaborations report new results on elastic VM crosssection and trajectory determination. Elastic VM production appears to be independent of the photon polarization. The presence of non-zero shrinkage in the photoproduction of $J / \psi$ indicates the presence of soft physics. Accumulated data do not accommodate a universal Pomeron trajectory. The steepness of the $x$ dependence of elastic VM production with changing $Q^{2}+M_{V}^{2}$ is similar to that of $F_{2}$ with changing $Q^{2}$.

\section{Introduction}

The production of vector mesons (VM, $V$ ) at HERA is interesting for the study of nonperturbative hadronic physics, perturbative QCD (pQCD) and their interplay, and the sensitivity to saturation effects. Moreover, VM production is complementary to deepinelastic scattering (DIS). Results from DIS have shown the correctness of pQCD down to low values of $Q^{2} \sim 1 \mathrm{GeV}^{2}$ (where $Q^{2}$ is the virtuality of the exchanged photon), a region where a transition to non-perturbative physics is observed [i] elastic processes were mostly treated through non-perturbative methods. These methods successfully describe the basic features of exclusive light VM production at low photon virtualities. However, in recent years a pQCD picture of the exclusive VM production is able to describe the basic features provided that $Q^{2} / \Lambda_{Q C D}^{2} \gg 1$ and $M_{V} / W \ll 1$, where $M_{V}$ is the mass of the $\mathrm{VM}$ and $W$ is the photon-proton center-of-mass energy. The photon fluctuates into a quark-antiquark pair, $q \bar{q}$, a color dipole, long before the interaction with the proton occurs. The interaction occurs via gluon ladders. The steepness of the rise of the VM production with $W$ is basically driven by the gluon density in the proton, which is probed at an effective scale, $\mu, \mu^{2} \sim\left(Q^{2}+M_{V}^{2}\right)$ at low $x\left(x \simeq\left(Q^{2}+M_{V}^{2}\right) / W^{2}\right)$.

Assuming a flavor independent production mechanism, the relative production rates should scale approximately with the square of the quark charges, i.e. the relative production rates scale as $\rho: \omega: \phi: J / \psi=9: 1: 2: 8$, referred to here as $\mathrm{SU}(4)$ ratios. It is interesting to determine how the $q \bar{q}-p$ scattering changes the $\mathrm{SU}(4)$ ratios.

\footnotetext{
${ }^{*}$ Speaker.
} 
As of today the H1 and ZEUS Collaborations ${ }^{1}$ have measured the elastic production of VM's, $e p \rightarrow e V p$, where $V=\rho, \omega, \phi$ and $J / \psi$ over a wide range of $W$ and from photoproduction $\left(Q^{2} \simeq 0\right)$ to $Q^{2}=100 \mathrm{GeV}^{2}$. The cross-section for $q \bar{q}$ scattering on the proton, $\sigma_{q \bar{q}}$, contains the dynamics with which the elastic VM production and total DIS cross-sections may be described. We are in a position to investigate the $x$ (or $W$ ) dependence of the VM production cross-section ordered according $Q^{2}+M_{V}^{2}$ and to investigate similarities between this and the $x$ dependence of the DIS total cross-section.

\section{Results}

The H1 Collaboration reports new results on the elastic photoproduction of $\rho$ mesons, where the high momentum proton is tagged in the forward proton spectrometer (FPS) [3i]. This experimental technique has the advantage that the elastic reaction is tagged directly. No subtraction of the proton dissociative production is needed. Additionally, by applying this method, one is able to measure directly the $t$ dependence of the cross-section, where $t$ is the square of the four-momentum transfer at the proton vertex. The kinematic range of the measurement is $25<W<70 \mathrm{GeV}, 0.073<|t|<0.4 \mathrm{GeV}^{2}$ and $E_{p^{\prime}} / E_{p}>0.98$, where $E_{p}$ and $E_{p^{\prime}}$ are the energy of the incoming and scattered proton, respectively.

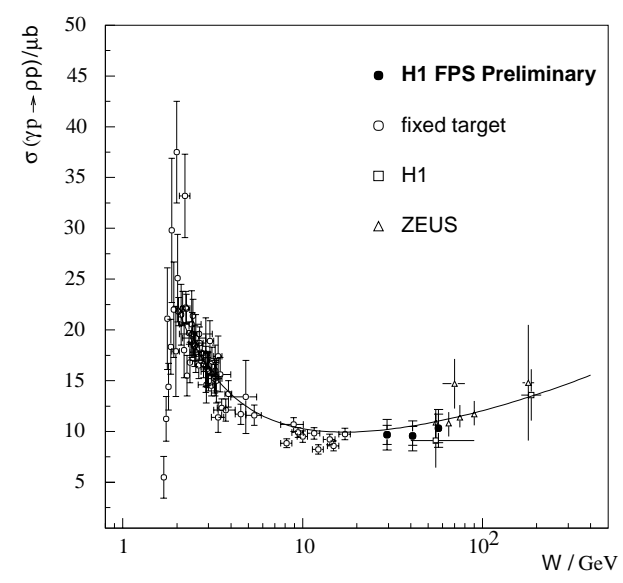

Figure 1: The elastic $\rho$ meson production cross-section $\sigma(\gamma p \rightarrow \rho p)$ compared with other measurements. The solid line corresponds to a fit by DL (see text).

The elastic cross-section is measured in three bins of $W$. The results are shown in Figure compared with other measurements performed at HERA and fixed target experiments, closing the gap in $W$ between them. The solid line corresponds to a fit performed on the energy dependence of the elastic scattering cross-sections in hadron-hadron collisions by Donnachie and Landshoff (DL) [4] Also, the slope of the differential cross-section, $b$, for $d \sigma / d t \propto \exp (b t)$ is measured as a function of $W$. The $t$ dependence of the Pomeron trajectory $\left(\alpha_{I P}(t)=\alpha_{I P}(0)+\alpha_{I P}^{\prime} t\right.$, where $\alpha_{I P}^{\prime}$ is also referred to as shrinkage) is com-

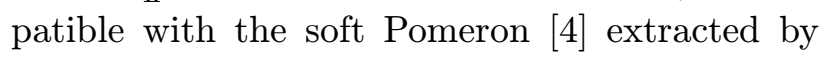
DL.

The ZEUS Collaboration reports new results on the electroproduction of $\rho$ mesons in the range $32<W<160 \mathrm{GeV}$ and for photon virtualities of $2<Q^{2}<80 \mathrm{GeV}^{2}$ 陭. The ratio of the production cross-section, $R=\sigma_{L} / \sigma_{R}$, for longitudinally $\left(\sigma_{L}\right)$ and transversely $\left(\sigma_{T}\right)$ polarized virtual photons is determined. The ratio increases with $Q^{2}$; however, it is constant with $W$. The Pomeron trajectory is extracted for the first time in the electroproduction regime from VM production (see Figure $\left.\overline{2}_{1}^{-1}\right)$. The trajectory parameters obtained in the range $2<Q^{2}<40 \mathrm{GeV}^{2}$ are $\alpha_{I P}(0)=1.14 \pm 0.01_{-0.03}^{+0.03}$ and $\alpha_{I P}^{\prime}=0.04 \pm 0.07_{-0.04}^{+0.13} \mathrm{GeV}^{-2}$.

\footnotetext{
${ }^{1} \mathrm{~A}$ compilation of references may be found in [2] collaborations are reviewed in the present contribution.
} 


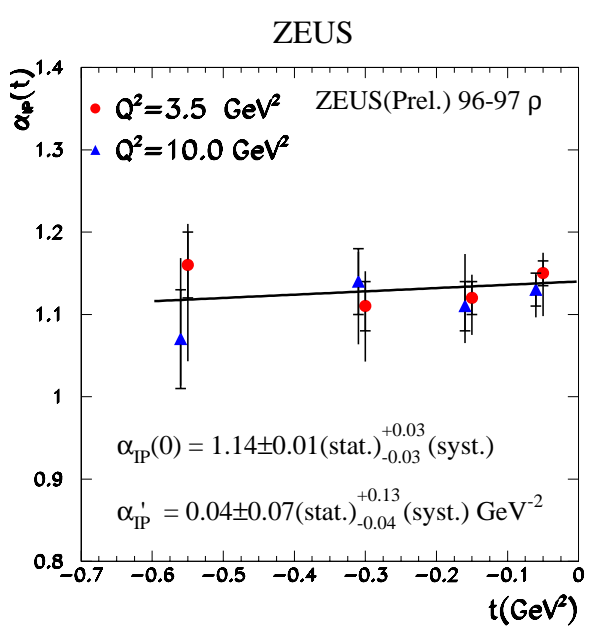

Figure 2: The Pomeron trajectory from the electroproduction of elastic $\rho$ as a function of $t$ for two $Q^{2}$ bins, $2<Q^{2}<6 \mathrm{GeV}$ and $6<$ $Q^{2}<40 \mathrm{GeV}^{2}$. The fit of the form $\alpha_{I P}(t)=$ $\alpha_{I P}(0)+\alpha_{I P}^{\prime} t$ is performed to all 8 points. The results of the fit are shown.

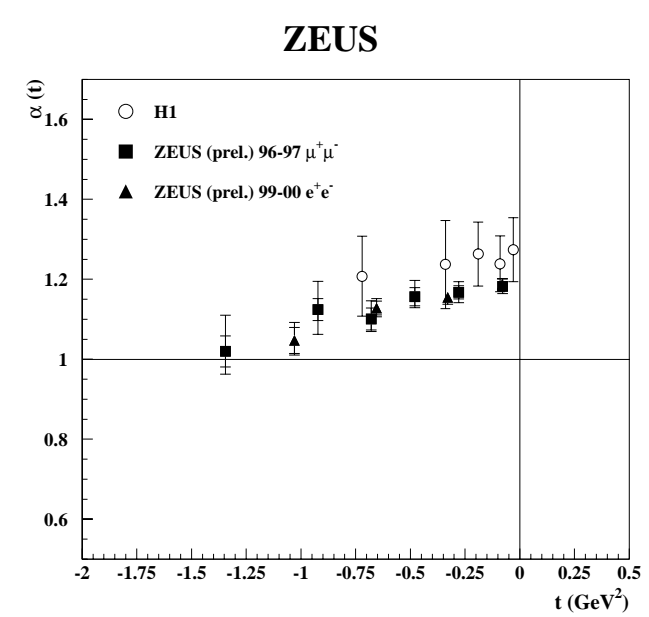

Figure 3: The Pomeron trajectory from the photoproduction of elastic $J / \psi$ as a function of $t$ from the H1 [6] and ZEUS experiments. The inner error bars indicate the statistical errors, the outer bars are the statistical and systematic uncertainties added in quadrature.

The ZEUS Collaboration reports new results on the differential cross-section, $d \sigma / d t$, of the photoproduction of $J / \psi$ in an extended range energy range $20<W<290 \mathrm{GeV}$ and $|t|<$ $1.25 \mathrm{GeV}^{2}$, with data corresponding to $55.3 \mathrm{pb}^{-1}$ of luminosity, using the electron decay channel [i]. The $t$-slope has been extracted in bins of $W$, yielding $b=4.3 \pm 0.08_{-0.41}^{+0.16} \mathrm{GeV}^{-2}$ at $W=90 \mathrm{GeV}$. The Pomeron trajectory has been extracted yielding $\alpha_{\mathbb{I P}}(0)=1.201 \pm$ $0.013_{-0.011}^{+0.003}$ and $\alpha_{I P}^{\prime}=0.126 \pm 0.029_{-0.028}^{+0.015} \mathrm{GeV}^{-2}$. The value of the latter parameter indicates the presence of small, but not negligible, shrinkage. This is illustrated in Figure

The H1 Collaboration presents results of the $t$-slope of the differential cross-section, $d \sigma / d t$, of the photoproduction of $\psi(2 S)$, studied through the leptonic decay channels [8ivil. The value of $b_{\psi(2 S)}=4.5 \pm 1.2_{-0.7}^{+1.4} \mathrm{GeV}^{2}$ is similar to that obtained in the photoproduction of $J / \psi$. The ZEUS Collaboration reports new results of the photoproduction cross-section of $\psi(2 S)$ studied through the electron decay channel in the range $50<W<125 \mathrm{GeV}\left[\overline{9_{1}}\right]$. The cross-section ratio $\sigma_{\gamma p \rightarrow \psi(2 S) p} / \sigma_{\gamma p \rightarrow J / \psi p}$ has been calculated. This result is consistent with previous measurements. Both results by the $\mathrm{H} 1$ and ZEUS Collaborations are consistent with the expectations of the quark parton model.

\section{Discussion}

The value of $\delta_{J / \psi} \approx 0.7$ for $\left(\sigma(\gamma p \rightarrow V p) \propto W^{\delta_{V}}\right)$ indicates a strong rise with $W$ for the cross-section of the photoproduction of $J / \psi$. This behavior is qualitatively different from that observed in the photoproduction of the $\rho$ meson, with the value of $\delta_{\rho} \approx 0.16$ [1] $\overline{0}_{1}^{\prime}$, [1]i]. This small value indicates that the production of $\rho$ mesons is dominated by soft physics. It is expected that the scattering of a transversely polarized $q \bar{q}$ state on proton is 
driven by a soft interaction. In the photoproduction regime, where the mean value of $Q^{2}$, $\left\langle Q^{2}\right\rangle \approx 5 \times 10^{-5} \mathrm{GeV}^{2} \ll M_{J / \psi}^{2}$, it is expected that, the $q \bar{q}$ state is transversely polarized ${ }^{2}$. Additionally, it has been observed that the value of $\delta_{J / \psi}$ does not increase with $Q^{2}$ in the electroproduction regime [i] $2 \overline{2}]$. This indicates that the $W$ dependence cross-section for longitudinally and transversely polarized $J / \psi$ are similar for the same final state. This is consistent with the observation that $R=\sigma_{L} / \sigma_{T}$ in the electroproduction of $\rho$ mesons is constant with $W$ [1] 3 in]. This leads to the conclusion that, despite the large differences in the dipole size expected for longitudinally and transversely polarized photons, the elastic production of VM's appears to be independent of the photon polarization.

Strong shrinkage is indicative of soft physics. In processes dominated by hard physics it is expected that $\alpha_{I P}^{\prime}$ is zero or very small. However, it has been observed that the value of $\alpha_{I P}^{\prime}$ obtained from the photoproduction of $J / \psi$ deviates from zero by some four standard deviations. It is not clear whether pQCD can accommodate this large value. This suggests that the interaction leading to the photoproduction of $J / \psi$ has a sizeable component of soft physics.

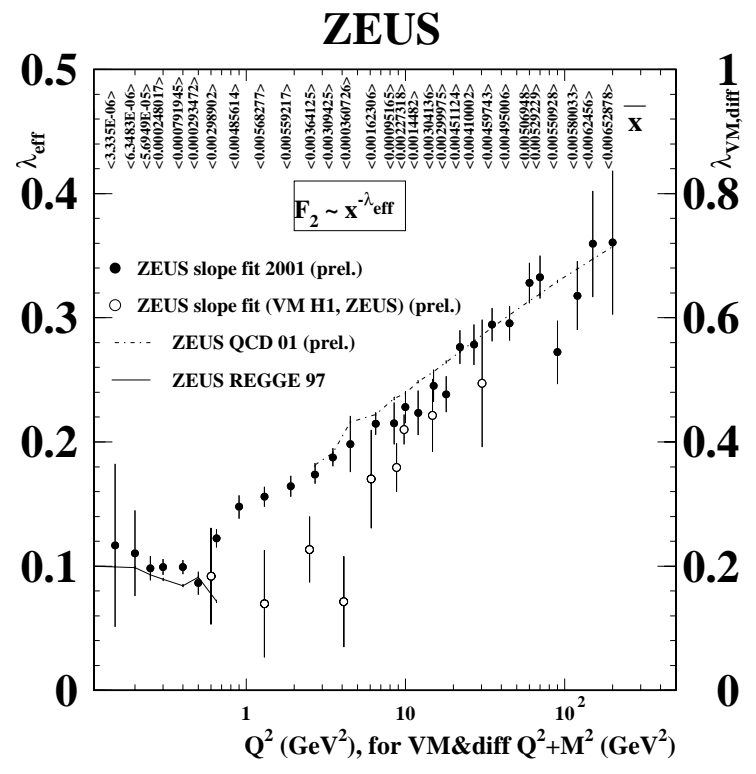

Figure 4: $\lambda_{V}$ and $\lambda_{\text {eff }}$ are compared (see text).
It has been shown that the $W$ and $t$ behaviors of the elastic VM production cross-sections do not depend strongly on the VM wave functions; the variable $Q^{2}+$ $M_{V}^{2}$ is a good choice of the scale of this

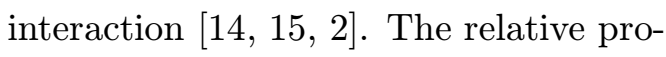
duction rate for different VM's may depend on the details of the wave functions. Data seem to indicate that the production rate of heavy VM's is enhanced with respect to the light VM's [i, īīi] after taking into account the $\mathrm{SU}(4)$ ratios.

The parameters of the Pomeron trajectory, have been extracted from a number of different VM's for different photon virtualities. The values of $\alpha_{I P}(0)$ show a clear dependence with $Q^{2}+M_{V}^{2}$. More data are needed to establish a clear

correlation between $\alpha_{I P}^{\prime}$ and $Q^{2}+M_{V}^{2}$. The data do not accommodate a universal Pomeron trajectory with fixed parameters.

Figure ${ }_{-1}^{1} \overline{4}$ shows the values of $\lambda_{V}\left(\left.\frac{d \sigma(\gamma p \rightarrow V p)}{d t}\right|_{t=0}\right.$ is fitted to $\left.A x^{-\lambda_{V}}\right)$ obtained for various VM's as a function of $Q^{2}+M_{V}^{2} \cdot \lambda_{V}$ is compared with $\lambda_{\text {eff }}$ obtained from the DIS total cross-section ${ }^{3}\left(F_{2} \propto x^{-\lambda_{e f f}}\right.$ at fixed $\left.Q^{2}\right)$. The behavior of the steepness of the rise of the VM production cross-section as $x \rightarrow 0$ with changing $Q^{2}+M_{V}^{2}$ is similar to the $x$

\footnotetext{
${ }^{2}$ The ratio of longitudinal to transversely polarized production of VM's is proportional to $Q^{2} / M_{V}^{2}$ for $Q^{2} \lesssim 1 \mathrm{GeV}^{2}$.

${ }^{3}$ Recent results of $F_{2}$ released by the ZEUS Collaboration [i]
} 
dependence of $F_{2}$ with changing $Q^{2}$. At small values of the scale $\left(Q^{2}\right.$ and $Q^{2}+M_{V}^{2}$ for DIS total and VM cross-sections, respectively) $\lambda_{\text {eff }}$ and $\lambda_{V}$ depend weakly on the scale. This correlation becomes stronger at larger scales. The similarities between $\lambda_{V}$ and $\lambda_{\text {eff }}$ are evident. This qualitative discussion serves as an indication that the dynamics that drive the increase of the DIS total and VM cross-sections are very similar. From the point

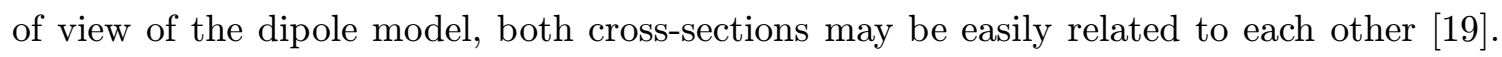
A theoretical description of $\sigma_{q \bar{q}}$ should be able to describe simultaneously both the DIS total and VM cross-sections. The addition of VM data to the analysis of $\sigma_{q \bar{q}}$ enhances the sensitivity of HERA data to issues like the interface between hard and soft physics and the sensitivity to saturation effects [20 $\left.{ }_{2}^{0}\right]$.

\section{References}

[1] ZEUS Collaboration, Eur. Phys. J. C7 (1999) 609.

[2] B. Mellado, "Diffractive VM Production at HERA", Proceedings of the ICHEP200, July 27 August 3 2000, Osaka, Japan, Volume I, 444.

[3] H1 Collaboration, Abstract 810, EPS01, July 12-18, 2001, Budapest, Hungary.

[4] A. Donnachie and P. V. Landshoff, Phys. Lett. B296 (1992) 227.

[5] ZEUS Collaboration, Abstract 594, EPS01, July 12-18, 2001, Budapest, Hungary.

[6] H1 Collaboration, Phys. Lett. B483 (2000) 23.

[7] ZEUS Collaboration, Abstract 548, EPS01, July 12-18, 2001, Budapest, Hungary.

[8] H1 Collaboration, Abstract 987, ICHEP2000, July 27 - August 3 2000, Osaka, Japan.

[9] ZEUS Collaboration, Abstract 562, EPS01, July 12-18, 2001, Budapest, Hungary.

[10] H1 Collaboration, Nucl. Phys. B463 (1996) 3.

[11] ZEUS Collaboration, Eur. Phys. J. C2 (1998) 247.

[12] ZEUS Collaboration, Abstract 879, July 27 - August 3 2000, Osaka, Japan.

[13] ZEUS Collaboration, Abstract 880, July 27 - August 3 2000, Osaka, Japan.

[14] B. Clerbaux, "VM Production at HERA", hep-ph/9908519.

[15] B. Naroska, "VM Production at HERA", Proceedings of XXXVth Rencontres de Moriond, les Arcs, France, February 2000, 360.

[16] ZEUS Collaboration, Addendum to Abstracts 548, 559, 594, EPS01, July 12-18, 2001, Budapest, Hungary.

[17] ZEUS Collaboration, Eur. Phys. J. C21 (2001) 3.

[18] See contribution from B. Surrow.

[19] A. Caldwell and M. S. Soares, "Vector Meson Production in the Golec-Biernat Wusthoff Model", hep-ph/0101085.

[20] See contributions from H. Kowalski and A. Gotsman. 